pond to thermal equilibrium ("white noise') then the particle cannot move on average in one direction ${ }^{9}$. In the presence of coloured noise the average flux depends strongly on the noise amplitude, in some cases showing a maximum for a well defined value ${ }^{7}$.

Earlier this year Doering et al. ${ }^{10}$ investigated how the average velocity of the particles depends on the characteristics of the out-of-equilibrium noise. Their combination of numerical and analytical results shows that not only the magnitude of the flux but also its sign depends on the nature of the noise. This means that for a given sawtooth potential the particles may be moved in either direction by simply changing the characteristics of time correlations in the nonthermal component of the noise.

Most of this theoretical activity was inspired not by Curie's old results but by developments in the study of motor enzymes $^{11}$. Molecular motors, such as kinesins or myosins, move on protein fibres many micrometres long, and can transport objects such as vesicles or chromosomes within the cell, participate in cell locomotion or accomplish other types of useful mechanical work. The energetics of the action of molecular motors has long been known ${ }^{12}$ : hydrolysis of bound nucleotides such as ATP provides the necessary energy. But the dynamics of this process is less well understood, although the crystal structure of muscle myosin has now been established ${ }^{13}$ and the action of single motors has been observed with impressive nanometre resolution $^{14,15}$. Not surprisingly, experimental data like these have inspired theorists to new efforts.

Motor enzymes and ratchets are indeed analogous in many respects. Both work in the presence of thermal noise and transport brownian particles; the ATP hydrolysis which drives a molecular motor is local, not involving gradients or forces on micrometre scales; the fibre on which a motor enzyme moves shows periodicity and spatial asymmetry because of the nature of its protein components; the binding sites for a motor on a fibre are analogous to the minima of a sawtooth potential; and the movement of a motor is directional, but different motors may move in different directions on the same fibre.

It is tempting to take the extra step and argue that a ratchet and a motor enzyme work in exactly the same way. Then one can try to compare quantitatively their characteristics? ${ }^{7}$. Unfortunately, one cannot then get anything beyond a simple dimensional analysis. The immediate problem is which of the ratchet models is the most appropriate. For instance, can the hydrolysis of ATP be considered as a source of a 'coloured', out-of-equilibrium noise which moves the motor in a fixed fibre potential? Or is it better to say that the motor enzyme can be found in two different states and ATP hydrolysis introduces nonthermal transitions between these states? One could also imagine a motor locally modifying the fibre on which it moves and thus changing the sawtooth potential, or some still more complicated situation.

The point is that all these questions are of microscopic nature and will be answered only by further experimental studies of the details of molecular motors. It is useful to know that simple physical devices can perform tasks analogous to those of biological systems, but this does not mean that the latter are as simple as the former.

Should we even try quantitatively to model the motor enzymes? Well, the effort might be worthwhile provided that one stays on the phenomenological rather than the microscopic level. For instance, in vitro motility assays ${ }^{16}$ characterizing the action of molecular motors tell us that the average motor velocity depends on ATP concentration, or on the load acting on the motors. Similarly, more quantitative motility assays might establish the relation between the mean hydrolysis rate and motor velocity, or correlations between the statistics of hydrolysis events and fluctuations in their movement. Phenomenological models explaining all these relations could also give some testable predictions, point to the differences in the action of different motors ${ }^{17}$ or even suggest some molecular (genetic) modifications which could alter the action of the motors.

Meanwhile, the work of Rousselet and her colleagues provides us with a beautiful example of biologically inspired physics and, in addition, a device with possible applications in biological research.

S. Leibler is in the Departments of Physics and of Molecular Biology. Princeton University, Princeton, New Jersey 08544, USA.

1. Rousselet, J., Saiome, L., Ajdari, A. \& Prost, J. Nature 370, 446-448 (1994)

2. Curie, P. J. Phys. III, 383 (1894)

3. Ajdari, A. \& Prost, J. C.R. Acad. Sci. Paris 315, 1635 $1639(1992)$

4. Feynman, R. P., Leighton, R. B. \& Sands, M. in The Feynman Lectures in Physics (Addison-Wesley, Reading. Massachusetts, 1966).

5. Volkmuth, W. D. \& Austin, R. H. Nature 358,600 (1992).

6. Magnasco. M. Phys. Rev. Lett. 71, 1477-1481(1993)

7. Astumian. R. D. \& Bier. M. Phys. Rev. Lett. 72, 17661769 (1994)

8. Prost, J., Chauwin, J.-F., Peliti, L. \& Ajdari, A. Phys. Rev. Lett. 72, 2652-2655 (1994)

9. Vale, R. \& Oosawa, F. Adv. Biophys. 26, 97-134 (1990)

10. Doering. C. R.. Horsthemke, W. \& Riodan, J. Phys. Rev. Lett. 72, 2984-2987 (1994).

11. Maddox, J. Nature 369, 181 (1994).

12. Hill, T. L. Thermodynamics for Chemists and Biologists (Addison-Wesley, Reading, Massachusetts, 1968)

13. Rayment, 1. et al. Science 261. 50-58 (1993).

14. Svoboda, K., Schmidt, C. F., Schnapp. B. J.\& Block. S. M. Nature 365, 721-727 (1993)

15. Finer, J. T. Simmons, R. M. \& Spudich. J. A. Nature $\mathbf{3 6 8}$ $113(1994)$.

16. Scholey, J. M. Motility Assays for Motor Proteins (Academic, New York, 1993).

17. Leibler, S. \& Huse, D. A. J. Cell Biol. 121, 1357 (1993).

\section{Radical solution}

AT last, some cheering news for smokers.

Dosing up with vitamin $\mathrm{C}$ can protect against one of smoking's nastier side effects - the clumping of white blood cells on vessel walls, which can trigger cardiovascular and pulmonary disease (H. A. Lehr, B. Frei and K. E. Arfors, Proc. natn. Acad. Sci. U.S.A. 91, 7688-7692; 1994). Vitamin C, which is a watersoluble vitamin, acts as an antioxidant, disarming poisonous oxygen radicals from cigarette smoke that cause the white blood cells to become sticky. But the water-insoluble vitamin $\mathrm{E}$, another antioxidant that works by a different mechanism, does not confer the same protection. The secret is probably that the oxygen species responsible for the effect on a smoker's leukocytes are more soluble in blood plasma.

\section{Old crocs}

CROCODILES today like their climate balmy -25 to $35^{\circ} \mathrm{C}$ is their preference, and anything above $39^{\circ} \mathrm{C}$ or below about $4{ }^{\circ} \mathrm{C}$ is inhospitable. So P. J. Markwick has been comparing the distributions of ancient crocodilians with their modern counterpart Alligator mississippiensis and drawing inferences about continental temperatures across North America in the Cenozoic era (Geology 22, 613-616; 1994). Fossil crocodilians from the Eocene (38-55 million years ago) are widespread, but by the late Oligocene the populations had apparently shrunk to little more than modern-day Florida. The Miocene again was crocodile-friendly, but the Pleistocene and early Holocene were emphatically not. The crocodilian lowdown on climate, part of a larger compilation of vertebrate records, thus backs up floral and modelling evidence for climes milder than today's during both Eocene and Miocene.

\section{High charges}

THE simplest of all atomic systems are those that, like the hydrogen atom, have just one electron and so avoid the complexities of multielectron interactions. And the higher the atomic number of an element, the greater the contributions of quantum electrodynamics and relativity to the atomic energy levels. The ideal testbed for theoretical models, then, is a heavy ion with every electron but one stripped away - such as the high-velocity $U^{91+}$ ions produced in a storage ring last year (T. Stohlker et al. Phys. Rev. Lett. 71, $2184 ; 1993)$. Now R. E. Marrs and coworkers have gone further by trapping single-electron $\mathrm{U}^{91+}$, and even a few naked $\mathrm{U}^{92+}$ ions, in a compressed electron beam (Phys. Rev. Lett. 72 , 4082-4085; 1994). With the ions more or less stationary, they were able to derive cross-sections for electron impact ionization, and suggest that current theoretical estimates are too low. 